\title{
Epidemiological Insights from 1,652 Patients with Spinal Tuberculosis Managed at a Single Center: A Retrospective Review of 5-Year Data
}

\author{
Bhavuk Garg, Nishank Mehta, Rudra Narayan Mukherjee, \\ Arun M. Swamy, Burhan S. Siamwala, Garima Malik \\ Department of Orthopaedics, All India Institute of Medical Sciences, New Delhi, India
}

\begin{abstract}
Study Design: Retrospective cohort.
Purpose: To report the demographic characteristics, clinico-radiological presentation, laboratory findings, and outcomes of "middlepath" treatment in patients with spinal tuberculosis from a single public healthcare facility in a developing country.

Overview of Literature: Tuberculosis is a global health problem that is endemic in developing countries and undergoing resurgence in developed ones. Spinal tuberculosis can cause disabling back pain, progressive deformity, and neurological involvement. However, there is a lack of large-scale epidemiological studies quantifying the size and severity of the problem of spinal tuberculosis.

Methods: Hospital records of spinal tuberculosis patients treated at a single center over a period of 5 years were retrospectively reviewed. A diagnosis of spinal tuberculosis was based on standard clinical, radiological, microbiological, and histopathological evidence. Patients were treated in accordance with the "middle-path" regimen; surgery was reserved for selective indications.

Results: A total of 1,652 patients were included. Their median age was 32.4 years, with $53 \%$ being male. Axial pain (98\%) was the most common presenting symptom; $19 \%$ of patients had neurological deficit. Lumbar spine (37\%) was the most common site of involvement, with a paradiscal pattern (82\%) of involvement predominating. Multi-level involvement was seen in $19 \%$ of patients; skip lesions were noted in 2.8\%. Transpedicular biopsy was performed in 667 patients; at least one tissue test was diagnostic of tuberculosis in $65 \%$ of patients. Forty-four patients had drug resistance to rifampicin. Surgery was required in $10.5 \%$ of patients. The "middle-path" regimen was associated with high compliance and significant improvements in pain (Visual Analog Scale score) and function (36-Item Short Form Health Survey).

Conclusions: Our findings confirm the widespread prevalence of spinal tuberculosis and describe various epidemiological characteristics of a large sample of spinal tuberculosis patients. Adoption of the "middle-path" regimen is associated with high compliance and favorable outcomes in spinal tuberculosis.
\end{abstract}

Keywords: Epidemiology; Infections; Pott's disease; Spinal tuberculosis; Tuberculosis

\section{Introduction}

With the advent of anti-tubercular chemotherapy and early radiographic diagnosis, it was believed that the eradication of tuberculosis (TB) would be possible. However, not only has TB survived, but it has thrown up new

Received Apr 7, 2021; Revised Jun 26, 2021; Accepted Jun 27, 2021

Corresponding author: Nishank Mehta

Department of Orthopaedics, All India Institute of Medical Sciences, Ansari Nagar, New Delhi, India

Tel: +91-9013370633, Fax: +91-1126594322, E-mail: mehta.nishank@gmail.com 
challenges such as drug resistance and co-infection with human immunodeficiency virus (HIV). Globally, more than 10 million people contracted TB in 2019 [1]. India has the highest share of TB cases, accounting for $26 \%$ of the global TB burden, which is 3 times the levels of the countries ranked second (Indonesia, 8.5\%) and third (China, 8.4\%) [1]. In 2019, a staggering 2.5 million new cases of TB were reported in India; in comparison, 8,920 TB cases were reported in the United States in the same year [2]. Given this context, any path to the global control or eradication of TB would inevitably pass through India. Likewise, the burden of extrapulmonary TB (ЕPTB) in India is high, accounting for approximately $16 \%$ of total new TB cases. This proportion is even higher among HIV-positive people [3]. Skeletal TB accounts for $10 \%$ of EPTB, with the spinal column alone accounting for half of skeletal TB cases [4]. While this may be only a fraction of the total TB burden, the ubiquity of TB in India makes spinal TB a diagnosis that is commonly encountered by orthopedic surgeons. Owing to its importance from a public health perspective, most of the country's resources for research, diagnosis, and treatment have conventionally been directed toward pulmonary TB. Nevertheless, spinal TB can cause significant ill health due to disabling back pain, progressive deformity, and neurological involvement [5].

The size and severity of the TB problem, along with its socioeconomic impact, suggest that the foundation for TB control has to be laid by large-scale public health programs. Achieving the control of TB requires a consolidated effort from various specialties, with each specialty battling a specific form of EPTB, within the framework of a larger war waged against TB. As spine surgeons, one of our major contributions would be to understand and report the demographic trends and epidemiological patterns of spinal TB, and to formulate and fine-tune treatment regimens that are appropriate in the context of resourceconstrained healthcare settings, where spinal TB is more common. There is a dearth of large-scale epidemiological studies focusing solely on spinal TB; understanding various epidemiological features of spinal TB can help in generating awareness among physicians, quantifying the scale of the problem, guiding resource allocation, and providing information regarding the prevalence of various clinical and radiological findings. Likewise, the management of spinal TB has also evolved from radical surgery to a more conservative "middle-path" approach, with the recogni- tion that uncomplicated spinal TB is essentially a "medical" disease [6,7]. However, there is still no global consensus over most aspects of treatment, such as the choice of antitubercular drugs, duration of chemotherapy, indications for surgery, and choice of surgical approach. The objectives of this study are: (1) to report on the demographic features, clinical presentation, radiographic presentation, and initial laboratory findings in a large sample of patients with spinal TB presenting to a single, tertiary-level, public healthcare facility in India, and (2) to describe various components of the "middle-path" regimen adopted at our center along with the outcomes of such a management approach.

\section{Materials and Methods}

The procedures used in this study adhere to the tenets of the Declaration of Helsinki. After approval from the Institutional Ethics Committee of All India Institute of Medical Sciences, New Delhi (IRB approval no., IEC-671/ RP-38/2020), data prospectively collected from the hospital records of patients who had received treatment for spinal TB at a single, tertiary-level, public healthcare facility in India between 2014 and 2018 were retrospectively analyzed. Patients signed informed consent regarding publishing their data and photographs. These included both patients who were operatively treated and those who were managed without surgical intervention. A diagnosis of spinal TB was ascribed to a patient in accordance with a composite reference standard, which required the fulfillment of one or more of the following criteria: (1) identification of Mycobacterium tuberculosis in one or more microbiological tests (growth on LowensteinJensen [LJ] solid culture, mycobacterial growth indicator tube [MGIT] liquid culture, acid-fast-positive staining on Ziehl-Neelsen [ZN] staining, Xpert MTB/RIF assay), (2) identification of typical histological features of TB (epithelioid granulomas, granulomatous inflammation, caseous necrosis, Langerhans giant cells) on histopathological examination (HPE), and (3) negativity in microbiological tests and HPE, but having clinical and radiological findings strongly suggestive of tubercular spondylodiscitis with a documented clinical, serological, and radiological response to empirical anti-tubercular treatment (ATT).

As part of the standard work-up in patients suspected or confirmed of having spinal $\mathrm{TB}$, the following investigations were performed in addition to detailed history- 
taking and physical examination: plain radiographs of the spine, chest radiograph, complete blood count, erythrocyte sedimentation rate (ESR), renal function test, liver function test (LFT), C-reactive protein (CRP), and wholespine magnetic resonance imaging (MRI). If a large psoas abscess was present, ultrasound-guided drainage with or without pigtail insertion was performed. Patients were screened for comorbid conditions and directed to appropriate specialty clinics for simultaneous management of these conditions. In approximately $40 \%$ of patients, we performed transpedicular biopsy under CT/fluoroscopy guidance. The biopsy samples were sent for histopathological and microbiological investigations (Xpert MTB, ZN staining, LJ/MGIT culture). Treatment was allocated in accordance with the "middle-path" regimen advocated by Tuli [6]. For rifampicin-sensitive patients, the total duration of anti-tubercular chemotherapy was 12 months, which comprised an intensive phase (4HRZE) of 4 months and a continuation phase (8HR) of 8 months. In rifampicin-resistant patients, second-line chemotherapy was started after consultation with an infectious disease specialist. Strict bed rest was not advised; patients remained ambulant, with an orthosis being prescribed for 3 months. Patients were followed up at monthly intervals during the intensive phase and at every 3 months thereafter until the completion of chemotherapy; a final followup visit was scheduled 6 months after the completion of chemotherapy to rule out disease relapse. Healing of the disease was documented by contrast-enhanced MRI performed after 12 months of chemotherapy, and correlating it with clinical improvement and normalization of ESR and CRP. Surgery was reserved for certain indications: no clinical or radiological response to treatment in 12 weeks, new-onset neurological deficit or worsening of existing neurological deficit on treatment, severe (Kumar and Tuli stage 3 or stage 4) paraplegia at onset, mechanical instability consequent to bony pathology causing back pain, and progressive or severe spinal deformity. Patients reported their Visual Analog Scale (VAS) scores and completed the 36-Item Short Form Health Survey (SF-36) questionnaire at stipulated follow-up intervals. The final outcome of treatment for each patient was graded into four categories (excellent, good, fair, and poor) based on a set of clinical, radiological, and functional criteria, as outlined in Table 1. Similar combinations of clinical, radiological, and functional criteria were also used in previous studies to grade final outcomes $[8,9]$.

Descriptive statistics were noted in the form of mean with standard deviation for normally distributed continuous data, median with range for continuous data not in a normal distribution, and frequency/percentage for categorical data. Changes in VAS score, SF-36 domain scores, ESR, and CRP at three different time-points (pre-chemotherapy, 3 months, and 12 months) were analyzed using

Table 1. Grading of outcome using clinical, radiological, and functional criteria

\begin{tabular}{|c|c|c|c|}
\hline \multirow{2}{*}{ Grade } & \multicolumn{3}{|c|}{ Criteria $^{\text {a) }}$} \\
\hline & Clinical & Radiological & Functional \\
\hline Excellent & $\begin{array}{l}\text { - Compliance to treatment } \\
\text { - No/minimal side effects to ATT } \\
\text { - Normalization of inflammatory markers at final follow-up }\end{array}$ & $\begin{array}{l}\text { - Complete radiological resolution of disease on contrast- } \\
\text { enhanced MRI and plain radiographs at } 12 \text {-month follow-up } \\
\text { - Post-treatment kyphotic angle }<30^{\circ} \text { (thoracic spine) and } \\
<20^{\circ} \text { (cervical and lumbar spine) }\end{array}$ & $\begin{array}{l}\text { - Improvement or no worsening of } \\
\text { pre-treatment neurological status } \\
\text { - 12-month follow-up VAS }<2\end{array}$ \\
\hline Good & $\begin{array}{l}\text { - Compliance to treatment } \\
\text { - Mild side effects to ATT not necessitating temporary ces- } \\
\text { sation/change of drugs, but needing additional medical } \\
\text { management } \\
\text { - Normalization of inflammatory markers at final follow-up }\end{array}$ & $\begin{array}{l}\text { - Complete radiological resolution of disease on contrast- } \\
\text { enhanced MRI and plain radiographs at } 12 \text {-month follow-up } \\
\text { - Post-treatment kyphotic angle }>30^{\circ} \text { (thoracic spine) and } \\
>20^{\circ} \text { (cervical and lumbar spine) }\end{array}$ & $\begin{array}{l}\text { - Improvement or no worsening of } \\
\text { pre-treatment neurological status } \\
\text { - 12-month follow-up VAS }<4\end{array}$ \\
\hline Fair & $\begin{array}{l}\text { - Non-compliance to treatment; treatment default } \\
\text { - Severe side effects to ATT necessitating temporary cessa- } \\
\text { tion/change of drugs } \\
\text { - Raised inflammatory markers at final follow-up }\end{array}$ & $\begin{array}{l}\text { - Incomplete radiological resolution of disease on contrast- } \\
\text { enhanced MRI and plain radiographs at 12-month follow-up }\end{array}$ & $\begin{array}{l}\text { - Improvement or no worsening of } \\
\text { pre-treatment neurological status } \\
\text { - 12-month follow-up VAS }>4\end{array}$ \\
\hline Poor & - Clinical deterioration necessitating surgical intervention & - Radiological deterioration necessitating surgical intervention & $\begin{array}{l}\text { - Worsening of neurological status } \\
\text { necessitating surgical intervention }\end{array}$ \\
\hline
\end{tabular}

ATT, anti-tubercular treatment; MRI, magnetic resonance imaging; VAS, Visual Analog Scale.

${ }^{\text {al } T h e ~ ' g r a d e ' ~ a s c r i b e d ~ w a s ~ i n ~ a c c o r d a n c e ~ w i t h ~ t h e ~ l o w e s t ~ o f ~ t h e ~ c l i n i c a l, ~ r a d i o l o g i c a l, ~ a n d ~ f u n c t i o n a l ~ c r i t e r i a ~ m e t ~ b y ~ t h e ~ p a t i e n t . ~ A ~ s i m i l a r ~ c o m b i n a t i o n ~ o f ~ c l i n i c a l, ~ r a d i o-~}$ logical, and functional criteria has been used to grade final outcomes in previous studies as well $[8,9]$. 
paired $t$-test or Wilcoxon's rank-sum test as applicable. A $p$-value $<0.05$ was considered to be statistically significant.

\section{Results}

Our study population comprised 1,652 patients, including 875 males (53\%) and 777 females (47\%). The median age was 32.4 years (range, 4-87 years), with the most commonly afflicted age group being $21-30$ years (33\%). Pediatric patients (age $<12$ years) accounted for $6.9 \%$, whereas geriatric patients (age $\geq 65$ years) accounted for $4.6 \%$ of the study population. One or more comorbidities were seen in 528/1,652 patients (32\%), with hypertension (11.8\%) and diabetes mellitus (9.2\%) being the most common. Overall, 19/1,652 patients (1.1\%) had HIV infection. Table 2 summarizes the baseline demographic characteristics of the study population.

Concomitant active pulmonary TB was seen in 69/1,652 patients $(4.1 \%)$, whereas a previous history of pulmonary TB was encountered in 101 patients $(6.1 \%)$. Six patients $(0.3 \%)$ had a previous history of EPTB: four patients had TB lymphadenitis and two had intestinal TB. Disseminated TB with spinal involvement was seen in four patients. The most common site of involvement in the spinal column was the lumbar spine $(611 / 1,652$ patients [37\%]) followed by the thoracic spine (439/1,652 patients [26.6\%]). The involvement of junctional areas of the spinal column was observed in 404/1,652 patients (24.5\%). The most common morphological pattern of involvement was paradiscal (1,354/1,652 patients [82\%]). Multi-level, contiguous involvement was present in 314/1,652 patients (19\%); this was determined to be present in patients who had more than one disc with its adjacent vertebrae involved if the morphology of the lesion was paradiscal. In patients with central or appendiceal morphology, the involvement of more than one contiguous vertebra was considered to be multi-level, contiguous involvement. Skip lesions/multifocal, non-contiguous involvement was seen in 46/1,652 patients $(2.8 \%)$. Table 3 summarizes the baseline radiological findings in the study population.

Back pain/neck pain (98\%) was overwhelmingly the most common symptom at presentation. Fewer patients presented with fever (33\%), loss of appetite $(22 \%)$, recent weight loss $(21 \%)$, radicular pain $(12 \%)$, and deformity (9\%). On average, patients had been suffering from one or more symptoms for $4.5 \pm 2.9$ months before presentation. Neurological involvement was seen in $314 / 1,652$ patients
(19\%), as graded by the Kumar and Tuli classification [10], $127 / 1,652$ patients $(7.7 \%)$ had stage 1 neurological involvement, 99/1,652 patients (6\%) had stage 2 neurological involvement, and 74/1,652 (4.4\%) patients had severe (stage 3 or 4) neurological involvement. A summary of the clinical findings in the study population is presented in Table 2.

Transpedicular biopsy from the lesion was performed in $667 / 1,652$ patients. Xpert MTB/RIF assay was positive in $367 / 667$ patients (55\%); 44 of these had rifampicin resistance. Positive ZN staining was noted in 37/667 patients (5.5\%). HPE was suggestive of TB in 267/667 patients (40\%). Growth on LJ culture was noted in only 19/667 patients (2.8\%). Combining all of the histopathological and microbiological investigations performed on the biopsy samples yielded sensitivity of $65 \%$ for the diagnosis of spinal TB; the biopsy was non-contributory in the rest of the cases. ESR was normal $(<20 \mathrm{~mm} / \mathrm{hr})$ in 365 patients (22.1\%), while serum CRP was normal ( $<10 \mathrm{mg} / \mathrm{L})$ in 455 patients (27.5\%). A summary of the findings from various laboratory investigations in the study population is presented in Table 4.

Data regarding the final outcome of management were available for 1,612/1,652 patients (Table 5). Overall, 37 patients were lost to follow-up, while three patients expired due to factors unrelated to spinal TB or its treatment. The rate of compliance with chemotherapy was 99\%; 16 patients turned into treatment-defaulters. Mild side effects of chemotherapy (nausea, mild arthralgia, gastrointestinal disturbance, generalized itching), which were successfully managed with additional medications without necessitating a change in the ATT, were seen in 212/1,612 patients (13\%). Moderate-to-severe side effects necessitating temporary cessation or modification of the ATT were seen in 66/1,612 patients (4\%). These included drug-induced hepatotoxicity $(\mathrm{n}=58)$, skin hypersensitivity reaction/dermatitis $(n=4)$, optic neuropathy $(n=3)$, and severe arthralgia $(n=1)$. Based on the indications for surgical treatment outlined earlier, 173/1,612 patients (10.7\%) required operative intervention. Severe neurological deficit at presentation was the most common indication for surgery; in these cases, patients were operated on without any delay. The average duration between diagnosis of spinal TB and surgical intervention in the remaining patients was 8.5 weeks. Significant improvements in mean VAS score and mean individual SF-36 domain scores were noted with the adoption of the "middle-path" regimen. 
Table 2. Summary of demographic and clinical characteristics of the study population $(n=1,652)$

\begin{tabular}{|c|c|}
\hline Characteristic & Value \\
\hline Age group (yr) & $32.4(4-87)$ \\
\hline$<10$ & $101(6.1)$ \\
\hline $11-20$ & $251(15.2)$ \\
\hline $21-30$ & $551(33.3)$ \\
\hline $31-40$ & $294(17.8)$ \\
\hline $41-50$ & $188(11.4)$ \\
\hline $51-60$ & $130(7.9)$ \\
\hline $61-70$ & $73(4.4)$ \\
\hline $71-80$ & $47(2.8)$ \\
\hline $81-90$ & $17(1.0)$ \\
\hline \multicolumn{2}{|l|}{ Sex } \\
\hline Male & $875(53.0)$ \\
\hline Female & $777(47.0)$ \\
\hline \multicolumn{2}{|l|}{ Comorbidities/co-existing medical conditions } \\
\hline Diabetes mellitus & $152(9.2)$ \\
\hline Hypertension & $195(11.8)$ \\
\hline Chronic liver disease & $44(2.7)$ \\
\hline Chronic kidney disease & $61(3.7)$ \\
\hline Coronary artery disease & $49(2.9)$ \\
\hline Cerebrovascular disease & $24(1.5)$ \\
\hline $\begin{array}{l}\text { Rheumatoid arthritis/ankylosing spondylitis/ } \\
\text { seronegative spondyloarthropathy }\end{array}$ & $20(1.2)$ \\
\hline HIV co-infection & $19(1.1)$ \\
\hline Others & $45(2.7)$ \\
\hline \multicolumn{2}{|l|}{ Associated pulmonary TB } \\
\hline Active & $69(4.1)$ \\
\hline Past history & $101(6.1)$ \\
\hline \multicolumn{2}{|l|}{ Associated extrapulmonary TB } \\
\hline Active & 4 \\
\hline Past history & 2 \\
\hline \multicolumn{2}{|l|}{ Symptoms } \\
\hline Pain (back/neck) & $1,620(98.1)$ \\
\hline Pain (radicular) & $196(11.9)$ \\
\hline Fever & $551(33.0)$ \\
\hline Weight loss & $344(20.8)$ \\
\hline Loss of appetite & $368(22.2)$ \\
\hline Deformity & $149(9.0)$ \\
\hline Mean duration of symptoms (mo) & $4.5 \pm 2.9$ \\
\hline \multicolumn{2}{|l|}{ Neurological status (Kumar-Tuli staging) } \\
\hline No deficit & $1,338(81.0)$ \\
\hline Stage-1 & $127(7.7)$ \\
\hline
\end{tabular}

(Continued on next page)
Table 2. Continued

\begin{tabular}{|c|c|}
\hline Characteristic & Value \\
\hline Stage-2 & $99(6.0)$ \\
\hline Stage-3 & $59(3.6)$ \\
\hline Stage-4 & $15(0.9)$ \\
\hline \multicolumn{2}{|l|}{ Indications for surgery ( $\mathrm{n}=173$ ) } \\
\hline Severe paraplegia at presentation & 74 \\
\hline Worsening neurological status while on ATT & 26 \\
\hline $\begin{array}{l}\text { Vertebral destruction/mechanical instability } \\
\text { causing intractable pain }\end{array}$ & 25 \\
\hline $\begin{array}{l}\text { Failure of response to conservative treatment } \\
\text { at } 12-16 \text { weeks }\end{array}$ & 20 \\
\hline Progressive deformity & 26 \\
\hline Uncertain diagnosis & 2 \\
\hline \multicolumn{2}{|l|}{ Surgical approach } \\
\hline Cervical $^{\text {a) }}$ & 22 (anterior) \\
\hline Cervicothoracic $^{\text {b) }}$ & 5 (anterior) \\
\hline Thoracic $^{c)}$ & $\begin{array}{c}1 \text { (anterior trans-thoracic) } \\
57 \text { (posterior) }\end{array}$ \\
\hline Thoracolumbar ${ }^{c)}$ & 19 (posterior) \\
\hline Lumbar $^{d l}$ & 58 (posterior) \\
\hline Lumbosacral $^{\text {dl }}$ & 11 (posterior) \\
\hline
\end{tabular}

Values are presented as median (range), number (\%), or mean \pm standard deviation.

HIV, human immunodeficiency virus; TB, tuberculosis; ATT, anti-tubercular treatment.

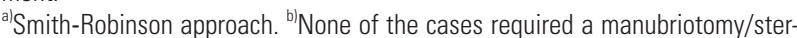
notomy. ${ }^{\mathrm{c}}$ Anterolateral decompression was done by a posterior midline incision by performing costotransversectomy/trans-facetal approach/trans-pedicular approach. ${ }^{\text {d) }}$ Anterolateral decompression was done by a posterior midline incision by a transforaminal approach/transverse process osteotomy.

This was accompanied by significant declines in the levels of inflammatory markers (ESR, CRP). Complete clinical and radiological resolution of the disease was observed at the end of 12 months of chemotherapy for 1,540/1,612 patients. In the remaining 72 patients, while an improvement was noted compared with the baseline findings, the ATT was continued for 18 months in view of persistent/ residual disease activity on contrast-enhanced MRI and raised inflammatory markers. Graded by the clinical-radiological-functional criteria outlined earlier, 1,363/1,612 patients (84.6\%) had an excellent or good outcome with the "middle-path" regimen, whereas a poor outcome was noted in $102 / 1,612$ patients (6.3\%). None of the patients had relapse of the disease at final follow-up. 
Table 3. Summary of imaging characteristics of the study population

\begin{tabular}{lc} 
Variable & \multicolumn{1}{l}{ Value } \\
\hline Regional distribution & $18(1.1)$ \\
\hline Craniocervical (occiput-C2) & $227(13.7)$ \\
\hline Cervical (C2/C3 disc-C6/C7 disc) & $18(1.1)$ \\
\hline Cervicothoracic (C7-T1/T2 disc) & $439(26.6)$ \\
\hline Thoracic (T2-T11) & $169(10.2)$ \\
\hline Thoracolumbar (T11/12 disc-L1) & $611(37.0)$ \\
\hline Lumbar (L1/L2 disc-L4/L5 disc) & $199(12.0)$ \\
\hline Lumbosacral (L5-S1) & $11(0.7)$ \\
\hline Sacrum (below S1) and coccyx & \\
\hline Morphology of lesion & $1,354(82.0)$ \\
\hline Paradiscal & $250(15.1)$ \\
\hline Central & $48(2.9)$ \\
\hline Appendiceal & \\
\hline No. of levels involved & $1,338(81.0)$ \\
\hline 1 & $199(12.0)$ \\
\hline 2 & $115(7.0)$ \\
\hline 23 & $46(2.8)$ \\
\hline Non-contiguous, multifocal involvement/skip lesions & \\
\hline
\end{tabular}

Values are presented as number (\%)

a) Upper thoracic (T2-T4)=54; middle thoracic (T4/5 disc-T8)=119; and lower

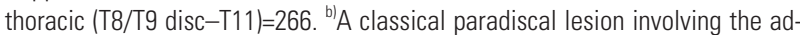
jacent end-plates of two contiguous vertebrae was considered as a single level involvement.

Table 4. Summary of laboratory findings in the study population

\begin{tabular}{lcc} 
Investigation & Positive & Negative \\
\hline LJ medium culture $^{\text {a) }}$ & $19(2.8)$ & $648(91.2)$ \\
$\begin{array}{l}\text { Presence of acid-fast bacilli on Ziehl Neelsen } \\
\text { staining }^{\text {a) }}\end{array}$ & $37(5.5)$ & $630(94.5)$ \\
Xpert TB assay $^{\text {a) }}$ & $367(55.0)$ & $300(45.0)$ \\
\hline Rifampicin resistance on Xpert TB assay & $44(\mathrm{MDR}+)$ & 323 \\
\hline Histopathological features suggestive of TB & $267(40.0)$ & $400(60.0)$ \\
\hline ESR $\leq 20 \mathrm{~mm} / \mathrm{hr}^{\mathrm{b})}$ & $365(22.0)$ & $1,287(78.0)$ \\
\hline CRP $\leq 5 \mathrm{mg} / \mathrm{L}^{\text {b) }}$ & $455(27.5)$ & $1,197(72.5)$ \\
\hline
\end{tabular}

Values are presented as number (\%).

TB, tuberculosis; MDR, multi-drug resistant; ESR, erythrocyte sedimentation rate; CRP, C-reactive protein.

a) Test was performed only in 667 patients who underwent transpedicular biopsy; percentages have been reported accordingly. ${ }^{b}$ Reference range for: ESR: $<10 \mathrm{~mm} / \mathrm{hr}$ (males), $<20 \mathrm{~mm} / \mathrm{hr}$ (females); CRP $\leq 5 \mathrm{mg} / \mathrm{L}$.

\section{Discussion}

Spinal TB is the most common form of osteo-articular
TB, accounting for $1 \%-5 \%$ of all TB cases $[11,12]$. While TB has declined in developed countries, it remains at large in the developing world. Owing to its designation as a global public health challenge, it is important to estimate the disease burden and study the demographic trends of TB. The WHO publishes the Global Tuberculosis Report every year and similar annual reports are published by individual countries; however, these quote consolidated figures for all TB cases. There is a dearth of large-scale epidemiological studies reporting the demographic, clinical, radiological, and laboratory findings in spinal TB. Most published studies are in the form of small case series with the study population comprising fewer than 100 patients. Considering that India harbors a quarter of the global TB burden, it is imperative that it takes the lead in undertaking such studies. Here, we report the demographic profile, clinico-radiological presentation, and initial laboratory findings in 1,652 patients who underwent treatment for spinal TB at a single center in India over a period of 5 years (2014-2018). In addition, we report the outcomes of the "middle-path" regimen for spinal TB followed at our center. The pioneering work of Tuli [6] in the "post-antitubercular drugs" era has made the indications of surgery in spinal TB selective; radical debridement has become a thing of the past. While such an approach has been wholeheartedly embraced in developing countries, routine surgery is still often practiced in developed countries, even for uncomplicated spinal TB, particularly with the increasing popularity of minimally invasive approaches. Most studies reporting on the outcome of conservative management of spinal TB have been limited by a small sample size or heterogeneity of data due to the involvement of multiple centers.

The earliest studies exclusively reporting on the epidemiological characteristics of patients with spinal TB in a large ( $>100$ patients) study population were published from France and Turkey, by Pertuiset et al. [13] and Turgut [14], respectively. A major shortcoming of these studies was that patients were treated at multiple centers, with a lack of uniformity in the data reported, the diagnostic approach adopted, or the surgical indications. In the decade that followed, two other multi-center studies-this time from developing countries in Africa-reported on the epidemiological characteristics of spinal TB patients $[15,16]$. The first large study from a single center was reported from China in 2012, involving a retrospective review of a total of 284 patients with spinal TB who were 
Table 5. Outcome measures at different time-points in the study population who completed final FU ( $n=1,612)$

\begin{tabular}{|c|c|c|c|c|c|}
\hline Outcome measure & At presentation & 3-mo FU & $p$-value $\mathrm{a}^{\mathrm{a})}$ & 12-mo FU & $p$-value ${ }^{\mathrm{b})}$ \\
\hline Visual Analog Scale & $8.2 \pm 2.9$ & $4.7 \pm 1.1$ & 0.045 & $1.1 \pm 0.8$ & 0.009 \\
\hline \multicolumn{6}{|l|}{ SF-36 } \\
\hline PF & $54.4 \pm 9.6$ & $62.6 \pm 9.6$ & 0.11 & $78.8 \pm 10.2$ & 0.023 \\
\hline $\mathrm{RP}$ & $38.6 \pm 7.6$ & $54.8 \pm 8.6$ & 0.048 & $70.7 \pm 11.7$ & 0.018 \\
\hline $\mathrm{BP}$ & $24.8 \pm 6.6$ & $45.6 \pm 7.8$ & 0.045 & $80.1 \pm 10.8$ & 0.008 \\
\hline $\mathrm{GH}$ & $58.6 \pm 8.0$ & $69.6 \pm 9.6$ & 0.19 & $80.2 \pm 12.2$ & 0.012 \\
\hline VT & $60.8 \pm 8.0$ & $69.9 \pm 10.1$ & 0.11 & $84.8 \pm 9.6$ & 0.033 \\
\hline SF & $48.5 \pm 7.6$ & $54.8 \pm 7.8$ & 0.22 & $75.5 \pm 9.7$ & 0.032 \\
\hline RE & $48.2 \pm 8.6$ & $64.8 \pm 9.7$ & 0.048 & $78.8 \pm 12.2$ & 0.021 \\
\hline $\mathrm{MH}$ & $55.8 \pm 8.7$ & $64.4 \pm 8.8$ & 0.09 & $78.8 \pm 11.4$ & 0.029 \\
\hline Erythrocyte sedimentation rate (mm/hr) & $54(4-124)$ & $42(1-78)$ & 0.11 & $12(1-36)$ & 0.02 \\
\hline C-reactive protein (mg/L) & $60(6-119)$ & $14(0.1-78)$ & 0.03 & $5(0.1-32)$ & 0.012 \\
\hline
\end{tabular}

Values are presented as mean \pm standard deviation or median (range). Overall outcome grading: excellent: 970 (60.2\%); good: 393 (24.4\%); fair: 147 (9.1\%); and poor: 102 (6.3\%). The SF-36 consists of eight domains: PF, RP, BP, GH, VT, SF, RE, and MH.

FU, follow-up; SF-36, 36-Item Short Form Health Survey; PF, physical functioning; RP, role-physical; BP, bodily pain; GH, general health; VT, vitality; SF, social functioning; $\mathrm{RE}$, role-emotional; $\mathrm{MH}$, mental health.

alRepresenting comparison of outcomes between "at presentation" and 3-month FU. ${ }^{b}$ Representing comparison of outcomes between "at presentation" and 12-month FU.

Table 6. Comparison of demographic characteristics of various studies reporting on epidemiology of spinal tuberculosis

\begin{tabular}{|c|c|c|c|c|c|c|c|}
\hline Authors (year) & Country & $\begin{array}{l}\text { Study } \\
\text { period }\end{array}$ & $\begin{array}{c}\text { No. of } \\
\text { hospitals }\end{array}$ & $\begin{array}{c}\text { No. } \\
\text { of patients }\end{array}$ & $\begin{array}{l}\text { Mean age } \\
(y r)\end{array}$ & $\begin{array}{c}\text { Sex ratio } \\
\text { (male:female) }\end{array}$ & $\begin{array}{l}\text { Time from symptom onset to } \\
\text { diagnosis (range) }\end{array}$ \\
\hline Pertuiset et al. [11] (1999) & France & 1980-1994 & 7 & 103 & 41 & $1.94: 1$ & 4 mo (1 wk-3 yr) \\
\hline Turgut [12] (2000) & Turkey & 1985-1996 & Multiple & 694 & 32.4 & $0.98: 1$ & NR \\
\hline Sakho et al. [13] (2003) & Senegal & 1986-1998 & 3 & 255 & 34.9 & $1.14: 1$ & 10.7 mo (1 mo-10 yr) \\
\hline Godlwana et al. [14] (2008) & South Africa & $2005-2006$ & 1 & 104 & NR & $1.36: 1$ & NR \\
\hline Wang et al. [15] (2012) & China & 2004-2010 & 1 & 284 & 38 & $1.07: 1$ & 18.0 mo (3 day-360 mo) \\
\hline Garza Ramos et al. [16] (2017) & USA & 2002-2011 & Multiple & 2,789 & 51 & $1.56: 1$ & NR \\
\hline Liu et al. [17] (2019) & China & 2007-2016 & Multiple & 1,378 & 43.7 & $1.40: 1$ & 16 mo (15 day-240 mo) \\
\hline Our study & India & 2014-2018 & 1 & 1,652 & 32.4 & $1.12: 1$ & $4.5 \mathrm{mo}$ (15 day-11 mo) \\
\hline
\end{tabular}

NR, not reported.

treated at a hospital in southwest China over a period of 7 years [17]. The mean age of the study population was 38.2 years, with nearly a third of patients being infected in the fourth decade of life (31-40 years). The study population was almost equally split between males and females (male:female ratio=1.07). Back pain $(92.6 \%)$ was the most common presenting symptom, while deformity $(28 \%)$, fever $(26 \%)$, and neurological deficit (21\%) were less frequent. Thoracic spine (45\%) was reported to be the most commonly affected region. Skip lesions were observed in $5.6 \%$ of patients, whereas $28 \%$ of patients had more than three contiguous vertebral bodies involved. MRI was not performed in all of the patients in this study, which may have compromised the accuracy of the radiological findings. Surprisingly, the authors reported the duration between symptom onset and diagnosis of the disease to be 18 months, with $43 \%$ of patients being diagnosed over a year after their symptom onset. In 2017, de la Garza Ramos et al. [18] reported on the epidemiology of 2,789 cases of spinal TB treated between 2002 and 2011 included in the Nationwide Inpatient Sample Database of the United States. A higher median age (51 years) and a 


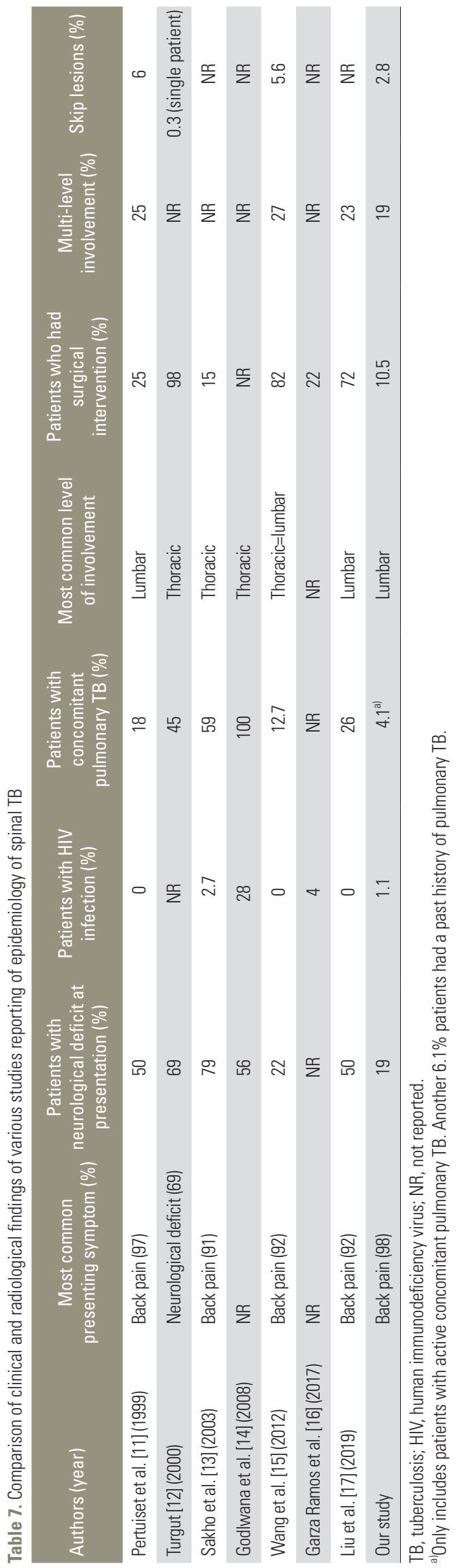

higher proportion of males (61\%) were reported in this study. Hypertension (28\%) was the most common associated comorbidity, followed by diabetes (11.6\%); overall, $4 \%$ of patients were HIV-positive. Surgery was performed in $619 / 2,789$ patients (22.2\%). Neither the presenting clinical features nor the radiological findings were reported. Another retrospective study reviewed the epidemiological profile of 1,378 spinal TB cases treated at various hospitals in south-central China between 2007 and 2016 [19]. The mean age of the study population was 43.7 years, with males accounting for $58 \%$ of the cases. Almost half of the patients had neurological impairment-this high proportion was possibly due to only in-hospital admissions being included. Lumbar spine (38\%), closely followed by thoracic spine (36\%), was the most commonly affected site. Concomitant pulmonary TB was present in $26.6 \%$ of patients. Surgical treatment was applied in 1,000/1,378 patients $(73 \%)$. Comparisons between the major findings in these studies and our study are presented in Tables 6 and 7.

To the best of our knowledge, our work is the first epidemiological study extensively reporting on the demographic, clinical, and radiological profile and noting the initial laboratory findings at presentation in a large sample of patients with spinal TB from India, the country with the highest TB burden. With all of the patients being recruited from a single center with a high volume of such cases, the diagnostic approach, management strategy, and operative indications remained standardized throughout the study period. The mean age of our study population was 32.4 years, with a third of patients being infected in the third decade of life (21-30 years). The lower mean age than in earlier studies is consistent with the much younger population of India. Geriatric patients (age $\geq 65$ years) accounted for $4.6 \%$ of the study population; the presence of comorbid conditions, a protracted and complicated course of illness, and risks of recumbency merit special attention to the management of these patients [12]. HIVpositivity was noted in only $1.1 \%$ of patients, which may be explained by the widespread social taboos associated with this condition, leading these patients to avoid mainstream medical care. Axial pain at the site of involvement was almost universally present (98\%); in comparison, constitutional symptoms such as fever (33\%), weight loss (21\%), and loss of appetite (22\%) were present less frequently. Unlike pulmonary TB, a clinician is more likely to encounter a patient with spinal TB with only a non- 
specific complaint of pain, rather than a more specific constellation of symptoms that stand out for TB. Despite the emphasis on public health awareness regarding TB, we observed that patients presented with neurological deficit (19\%) and deformity (9\%), and noted a mean delay of 4.5 months between symptom onset and diagnosis. A dedicated information drive to raise awareness of spinal TB may help in improving these figures. The most commonly affected region of the spinal column in our study was the lumbar spine (37\%), which contrasts with previous studies reporting that the thoracic spine was the most commonly involved site. Junctional areas of the spine are biomechanically critical due to their predisposition to instability and progressive deformity. In contrast to earlier studies, we noted that the lumbosacral junction (12\%) was more commonly affected than the thoracolumbar junction (10\%). Despite performing whole-spine MRI in all of our patients, the proportions of patients with multi-level, contiguous involvement (19\%) and skip lesions (2.8\%) were similar to those noted in earlier studies. Owing to the high case burden and logistic limitations, we could not perform a transpedicular biopsy in all of the patients.

However, among the 667 patients who underwent a biopsy, an overall diagnostic yield of $65 \%$ was observed. The paucibacillary nature of spinal TB lesions may have led to the biopsy being non-contributory in over a third of patients. Operative intervention was necessary in 173/1,652 patients (10.5\%), a much smaller proportion than in earlier studies. This can be ascribed to the widespread adoption of the "middle-path" regimen in India. Most of the patients in our study were treated with ambulant chemotherapy; in contrast, earlier studies by de la Garza Ramos et al. [18] and Liu et al. [19] only included inpatient hospital admissions, which were more likely to be operated upon. The "middle-path" regimen adopted at our hospital comprised ambulant chemotherapy for a duration of 12 months. This longer duration of treatment including a 4-month intensive phase was applied in consideration of the poor penetration of anti-tubercular drugs in spinal lesions and the difficulty in assessing healing response in spinal TB. However, a recent study reported no difference in healing response or relapse rate with short-course (6 months) chemotherapy [20]. We observed a high compliance rate $(99 \%)$ and a low incidence of side effects of antitubercular drugs. Serial monitoring via LFTs and more frequent follow-ups during the intensive phase can aid the early detection of drug-induced hepatotoxicity, which was the most commonly encountered side effect necessitating a change in drug regimen. Using composite clinical, radiological, and functional criteria to define the outcome of management, $84.5 \%$ of patients had an excellent-to-good outcome with the "middle-path" regimen. In comparison, $6.3 \%$ of patients, largely comprising those who had to undergo surgery for preventing or addressing mechanical or neurological complications, had a poor outcome. Mean VAS scores and CRP levels showed significant improvement as early as 3 months after the institution of chemotherapy, whereas all individual SF-36 domain scores showed significant improvement at 12-month followup. For thoracic, thoracolumbar, and lumbar spine, the posterior approach allows global access, adequate ventral decompression of the spinal cord, and anterior column reconstruction by rib autograft or cage. This has largely obviated the need for the more morbid anterior transthoracic or retroperitoneal approach in our practice. Our experience with the "middle-path" regimen in a larger study population echoes the findings of earlier studies reporting success with ambulant chemotherapy and selective surgery for spinal TB, even in cases with mild (grade 1 or 2) neurological deficit, albeit with smaller sample sizes [7,8,21-23].

We recognize certain limitations in our study, with its retrospective nature being the foremost among them. The robustness of the data presented was reliant on hospital records analyzed retrospectively. In addition, all patients were recruited from a single center. The inclusion of patients from multiple centers would strengthen the observations, provided that there is uniformity in the diagnostic approach, decision-making, and treatment indications. Transpedicular biopsy was not performed in all patients, so the prevalence of drug-resistant TB in our study population could not be estimated. A robust notification and information management system is the bedrock of a successful program to control TB. While the focus of governments and public health organizations has been on consolidated the reporting and surveillance of TB cases, there is a lack of literature focusing solely on spinal $\mathrm{TB}$, possibly due to the low incidence of this condition in developed countries. With real-time, paperless, electronic health record systems being embraced by most countries, it is important to undertake epidemiological studies that can serve as a template for data collection. Simultaneously, well-defined treatment protocols, based on the "middlepath" philosophy, should be in place. 


\section{Conclusions}

The findings of our study confirm that spinal TB is widespread in India, with more than 300 spinal TB patients seeking treatment at our hospital annually. The disease most commonly affects young adults, with most patients presenting only pain - a rather non-specific symptomafter an average delay of 4.5 months. In the vast majority of patients, operative intervention is not needed. With increasing prevalence of drug resistance, it is advisable to seek a microbiological or histopathological confirmation of diagnosis in all cases before instituting first-line ATT; our findings suggest that biopsy may not be contributory, and one may need to rely on clinical and radiological findings while being watchful for a response to ATT. Treatment protocols based on the "middle-path" philosophy are associated with favorable clinical, radiological, and functional outcomes, largely mitigating the need for routine surgery in spinal TB. Large-scale, prospective, multi-center epidemiological studies, preferably from geographically, ethnically, and socioeconomically diverse study sites, should be undertaken to understand where we stand today in our fight against spinal TB.

\section{Conflict of Interest}

No potential conflict of interest relevant to this article was reported.

\section{References}

1. World Health Organization. Global tuberculosis report 2020. Geneva: World Health Organization; 2020.

2. Schwartz NG, Price SF, Pratt RH, Langer AJ. Tuberculosis: United States, 2019. MMWR Morb Mortal Wkly Rep 2020;69:286-9.

3. World Health Organization. Tuberculosis control in the South-East Asia region: annual TB report 2014. New Delhi: WHO Regional Office for South-East Asia; 2014.

4. Rajasekaran S, Soundararajan DC, Shetty AP, Kanna RM. Spinal tuberculosis: current concepts. Global Spine J 2018;8(4 Suppl):96S-108S.

5. Sharma SK, Ryan H, Khaparde S, et al. Index-TB guidelines: guidelines on extrapulmonary tuberculosis for India. Indian J Med Res 2017;145:448-63.
6. Tuli SM. Results of treatment of spinal tuberculosis by "middle-path" regime. J Bone Joint Surg Br 1975;57:13-23.

7. Bakhsh A. Medical management of spinal tuberculosis: an experience from Pakistan. Spine (Phila Pa 1976) 2010;35:E787-91.

8. Nene A, Bhojraj S. Results of nonsurgical treatment of thoracic spinal tuberculosis in adults. Spine J 2005;5:79-84.

9. Valsalan R, Purushothaman R, Raveendran M, Zacharia B, Surendran S. Efficacy of directly observed treatment short-course intermittent regimen in spinal tuberculosis. Indian J Orthop 2012;46:138-44.

10. Jain AK, Kumar S, Tuli SM. Tuberculosis of spine (C1 to D4). Spinal Cord 1999;37:362-9.

11. Tuli SM. General principles of osteoarticular tuberculosis. Clin Orthop Relat Res 2002;(398):11-9.

12. Fuentes Ferrer M, Gutierrez Torres L, Ayala Ramirez O, Rumayor Zarzuelo M, del Prado Gonzalez N. Tuberculosis of the spine: a systematic review of case series. Int Orthop 2012;36:221-31.

13. Pertuiset E, Beaudreuil J, Liote F, et al. Spinal tuberculosis in adults: a study of 103 cases in a developed country, 1980-1994. Medicine (Baltimore) 1999;78:309-20.

14. Turgut M. Spinal tuberculosis (Pott's disease): its clinical presentation, surgical management, and outcome: a survey study on 694 patients. Neurosurg Rev 2001;24:8-13.

15. Sakho Y, Badiane SB, N'Dao AK, N'Diaye A, Gueye M, N'Diaye IP. Pott's disease in Senegal. Eur J Orthop Surg Traumatol 2003;13:13-20.

16. Godlwana L, Gounden P, Ngubo P, Nsibande T, Nyawo K, Puckree T. Incidence and profile of spinal tuberculosis in patients at the only public hospital admitting such patients in KwaZulu-Natal. Spinal Cord 2008;46:372-4.

17. Wang H, Li C, Wang J, Zhang Z, Zhou Y. Characteristics of patients with spinal tuberculosis: seven-year experience of a teaching hospital in Southwest China. Int Orthop 2012;36:1429-34.

18. De la Garza Ramos R, Goodwin CR, Abu-Bonsrah N, et al. The epidemiology of spinal tuberculosis in the United States: an analysis of 2002-2011 data. J Neurosurg Spine 2017;26:507-12.

19. Liu Z, Wang J, Chen GZ, et al. Clinical characteristics of 1378 inpatients with spinal tuberculosis in general 
hospitals in South-Central China. Biomed Res Int 2019;2019:9765253.

20. Nene AM, Patil S, Kathare AP, Nagad P, Nene A, Kapadia F. Six versus 12 months of anti tubercular therapy in patients with biopsy proven spinal tuberculosis: a single center, open labeled, prospective randomized clinical trial: a pilot study. Spine (Phila Pa 1976) 2019;44:E1-6.

21. Moon MS, Moon YW, Moon JL, Kim SS, Sun DH. Conservative treatment of tuberculosis of the lumbar and lumbosacral spine. Clin Orthop Relat Res 2002;(398):40-9.
22. Wimmer C, Ogon M, Sterzinger W, Landauer F, Stockl B. Conservative treatment of tuberculous spondylitis: a long-term follow-up study. J Spinal Disord 1997;10:417-9.

23. Kotil K, Alan MS, Bilge T. Medical management of Pott disease in the thoracic and lumbar spine: a prospective clinical study. J Neurosurg Spine 2007;6:2228. 